\title{
Cell-phone use diminishes self-awareness of impaired driving
}

\author{
David M. Sanbonmatsu ${ }^{1}$ • David L. Strayer ${ }^{1}$. Francesco Biondi ${ }^{1}$ Arwen A. Behrends ${ }^{1}$. \\ Shannon M. Moore ${ }^{1}$
}

Published online: 18 August 2015

(C) Psychonomic Society, Inc. 2015

\begin{abstract}
Multitasking diminishes the self-awareness of performance that is often essential for self-regulation and selfknowledge. Participants drove in a simulator while either talking or not talking on a hands-free cell phone. Following previous research, participants who talked on a cell phone made more serious driving errors than control participants who did not use a phone while driving. Control participants' assessments of the safeness of their driving and general ability to drive safely while distracted were negatively correlated with the actual number of errors made when they were driving. By contrast, cell-phone participants' assessments of the safeness of their driving and confidence in their driving abilities were uncorrelated with their actual errors. Thus, talking on a cell phone not only diminished the safeness of participants' driving, it diminished their awareness of the safeness of their driving.
\end{abstract}

Keywords Multi-tasking · Self-regulation · Self-awareness · Distracted driving

A process that is often central to the pursuit and attainment of goals is monitoring (Baumeister, Heatherton, \& Tice, 1994). In the monitoring of an activity, individuals track their thoughts, feelings, behavior, and circumstances, and their progress toward their goals. When discrepancies are perceived between goals or standards, and performance, action may be

David M. Sanbonmatsu

sanbonmatsu@psych.utah.edu

$\triangle$ David L. Strayer

david.strayer@utah.edu

1 Department of Psychology, University of Utah, 380 S. 1530 E. RM. 502, Salt Lake City, UT 84112-0251, USA taken to bring the self into line with those standards (Carver \& Scheier, 1998). The self-awareness characterizing monitoring also facilitates self-knowledge; by observing themselves, individuals can gain a better understanding of their skills, abilities, and preferences. When monitoring is lacking, individuals may not recognize the shortcomings of their performance, and, hence, may fail to take immediate corrective action. They may also fail to learn about themselves, which may hamper their ability to make sound task-related decisions in the future (Moore, Behrends, Mazur, \& Sanbonmatsu, 2014).

Factors that draw attention away from the self and diminish monitoring may contribute to self-regulatory failure (e.g., Baumeister, Heatherton, \& Tice, 1994). Thus, studies have shown that the ability to monitor may be impaired by strong emotions or drives (Sayette, Schooler, \& Reichle, 2010). Researchers have speculated that self-awareness may be similarly affected by alcohol intoxication and other conditions that limit the ability to process information (e.g., Sayette \& Griffin, 2011).

The monitoring of a particular activity may also be impaired by multitasking. Multitasking involves the concurrent performance of two or more functionally independent attention-demanding tasks having unique goals and involving distinct stimuli, mental transformation, and response outputs. People often multitask to get more done and to experience more activities (Sanbonmatsu et al., 2013). However, multitasking can be cognitively and physically taxing. Moreover, performance of the tasks often suffers such that errors are made and overall productivity is diminished (Crenshaw, 2008). The attentional demands of each task as well as the inhibitory requirements of task switching may also diminish task monitoring; because attention is distributed across multiple tasks, the attention given to the self and the task context may be limited. Consequently, individuals may be less apt to notice the ways in which their performance is falling short and 
less likely to make compensatory adjustments. Moreover, because their self-awareness is diminished, they may be less able to acquire knowledge of their skills and abilities, including their general ability to multitask. This may foster persistent misperceptions about their personal ability to multitask. Indeed, prior research has shown that people are overconfident about their ability to multitask effectively and that their selfassessments are largely unrelated to their actual multitasking abilities (Sanbonmatsu et al., 2013).

\section{Multitasking behind the wheel}

An important applied context in which the self-regulatory failures associated with multitasking may occur is in the operation of a motor vehicle. When people are driving, they often engage in secondary tasks such as eating and grooming. Indeed, one of the most common multitasking activities behind the wheel is talking on a cell phone. Estimates suggest that at any daylight hour $10 \%$ of all drivers on the road are talking on their cell phone (e.g., Glassbrenner, 2005). This is a public safety issue because of the heightened crash risk associated with cell-phone usage (e.g., McEvoy et al., 2005; Redelmeier \& Tibshirani, 1997) and the substantial body of empirical evidence showing the impairments from talking on a cell phone (Strayer \& Drews, 2007; Strayer, Watson, \& Drews, 2011).

Recent work (Sanbonmatsu et al., 2015) indicates that drivers are motivated to use cell phones by a variety of perceived benefits. Talking on a cell phone enables drivers to connect with family and friends, get work done, and alleviate the boredom associated with driving. Although people generally acknowledge the risks, they downplay the dangers of talking on a cell phone relative to drinking and driving. Most actually believe they can drive safely while using a cell phone and regard themselves to be more capable than others of driving while distracted. Our findings indicate that this overconfidence is one of the strongest predictors of drivers' usage of cell phones behind the wheel.

An important reason why drivers may be overconfident about their ability to drive safely while distracted and why people more generally overestimate their ability to multitask effectively is diminished monitoring. Drivers generally monitor their performance to ensure that they are driving safely. However, when they are distracted by a cell phone, they may be less cognizant of the errors they are making and how poorly they are driving. Because of their lack of self-awareness, they may maintain the illusion they can drive safely while talking on the cell phone and continue engaging in this risky behavior. Moreover, because their ability to monitor their driving is impaired, their performance assessments are likely to be guided by their beliefs and expectations rather than actual observations.
We conducted a study to examine the impact of multitasking on performance monitoring and assessment. We hypothesized that engagement in multiple tasks diminishes awareness of task performance and the accuracy of self-performance ratings. Because self-awareness of the costs of multitasking is limited, overconfidence may prevail. These effects of multitasking were investigated in the ubiquitous context of operating a motor vehicle. Participants drove in a simulator while either (a) concurrently talking or (b) not talking on a cell phone. An experimenter recorded driving errors such as running a red light and swerving into the oncoming lane. The errors served as the measure of the actual safeness of participants' driving. Participants' self-awareness of their driving safety was measured in two ways. First, they rated the safeness of their simulator driving. Second, they attempted to remember the specific driving errors they made. Participants finished by indicating their general confidence in their ability to drive safely while distracted.

Following prior research, cell phone use was expected to increase the driving errors made by participants. More importantly, cell phone use was expected to diminish the accuracy of participants' assessments of the safeness of their driving. We also predicted that control participants who were not using a cell phone would have better memory of their errors than participants talking on a cell phone. Finally, control participants' confidence in their ability to drive safely while distracted was expected to be grounded in the actual safeness of their driving. However, because cell phone users were expected to be less aware of the adverse effects of cell phone use on their driving, it was anticipated that their assessments of their general ability and their errors would be uncorrelated.

\section{Method}

\section{Participants}

One hundred undergraduates (67 females and 33 males) participated in the Institutional Review Board-approved study for extra course credit. They were randomly assigned to either the cell-phone group or a control group that did not use a cell phone. The undergraduates ranged in age from 18 to 41 years, with an average age of 21.8 years. All participants owned a cell phone. They reported that they drove an average of 70 min each day $(\mathrm{SE}=9)$, and used their cell phone approximately $22 \%(\mathrm{SE}=2.1)$ of the time that they were driving.

A power analysis for detecting driving differences between the cell-phone and control groups was performed adopting an $\alpha$ of .05 (2-tailed). Following our previous research (e.g., Strayer, Drews, \& Johnston, 2003), we assumed a moderate to large effect size. The analysis indicated that a sample of 30 participants in each condition was sufficient to demonstrate 
differences in driving performance. A second power analysis was performed for the planned correlational analyses of the correspondence between driving performance and assessment. Assuming a moderate to large effect size and adopting an $\alpha$ of .05 (2-tailed), we determined that 50 participants in each condition were needed. Note that individuals who were not able to complete the driving session because of motion sickness were not included in the participant count.

\section{Stimuli and apparatuses}

The DriveSafety ${ }^{\mathrm{TM}}$ DS-600 simulator was used in this experiment. The DS-600 consists of a Ford Focus cab surrounded by three large screens encompassing a $270^{\circ}$ view. The simulated vehicle is based on the vehicular dynamics of a compact passenger sedan with automatic transmission.

The driving scenario was designed using DriveSafety HyperDrive Authoring Suite. A city residential road database simulated an 8.2-km section of road with ten intersections and both one-way and two-way traffic. Directional arrows embedded in the driving environment provided instructions for navigation. Driving was regulated by speed limit, school zone, stop and yield signs, and traffic lights. The roadways, sign placement (e.g., distance between the crosswalk and the pedestrian crossing sign), and crosswalks conformed to the Manual on Uniform Traffic Control Devices for Streets and Highways (Federal Highway Administration, 2012).

Participants in the cell-phone group used a hands-free device provided by the experimenter. Cellular service was provided by Sprint. The cell phone was an iPhone 5 s running iOS 7 and the hands-free earpiece was manufactured by Jawbone. Participants selected which ear they wished to use for the hands-free earpiece.

\section{Procedure}

Participants began the study by completing a brief survey of their driving practices and beliefs. They were then familiarized with the driving simulator, using a standardized adaptation sequence designed to reduce the likelihood of motion sickness, after which commenced a 10-min practice session. After completing the survey, cell-phone participants were asked to generate a list of friends or family members they could talk with during their drive. The call was initiated and the volume was adjusted before they began the primary driving session.

There were 12 potentially hazardous driving scenarios or situations that were encountered by participant drivers. Specifically, drivers were required to stop for pedestrians at a crosswalk partially obscured by other vehicles, slow down in a school zone, yield to an approaching vehicle, stop behind cars turning left while traveling on a single lane road, slow down and move over because of construction, avoid a bicyclist at a crosswalk, pull over for an ambulance, and slow down for a crosswalk partially obscured by a truck. The entire 8.2-km drive took about $20 \mathrm{~min}$ to complete.

The experimenter observed the performance of the driver from behind the simulator cab. As the participant drove, the experimenter tabulated the driving errors on a checklist. The specific errors that were recorded on the checklist are presented in Table 1. The total number of each type of driving error was tabulated by the experimenter. Immediately after completing their drive, participants were asked to complete the same checklist of errors that the experimenter had filled out. Thus, they attempted to recall the number of driving errors of each type they had made on the simulator.

Participants finished by assessing the safeness of their driving, and their general ability to drive safely while talking on a cell phone. They rated "the safeness of your driving during this experiment using the simulator" on a 7-point scale anchored by " $-3 "=$ not at all safe and "+3" = highly safe. Finally, they answered the question: "To what extent are you capable of driving safely while engaging in another task such as talking on the cell phone?" on a 7-point scale anchored by " $1 "=$ not at all capable and "7" = highly capable.

\section{Results}

The analyses were limited to participants who completed the simulator drive or whose drive was not interrupted. Preliminary analyses indicated that gender did not interact with cellphone usage to affect any of the performance or assessment measures in the study. Consequently, gender was not included in the reported analyses.

\section{Driving errors and driving safeness}

The most basic measure of the actual safeness of participants' driving on the simulator was the total number of errors they made. In order to distinguish the severity of the errors and create more detailed measures of driving safeness, we asked a different group of 69 respondents to indicate the seriousness of each of the driving errors or violations on a 6-point scale anchored by " $0 "=$ not at all serious and " $5 "=$ highly serious. The mean rating of each of the driving errors is presented in Table 1. There were two errors (failing to signal and speeding) that were perceived to be less severe than the others. When the errors were ordered in terms of judged severity, the only significant pair-wise contrast between an error and the next most serious error was between the errors of speeding and making an illegal turn, $t(68)=2.17, p=.033, d=.53$. Consequently, failing to signal and speeding were labeled as "minor" errors while the remaining ten were labeled as "serious" errors. The minor errors have face validity as being less severe in that they are less likely to directly lead to an accident than more serious 
Table 1 Driving errors and their ratings of severity $(\mathrm{N}=69)$

\begin{tabular}{ll}
\hline & Mean \\
\hline Failing to signal a turn & $2.51[2.20-2.81]$ \\
Speeding $10 \mathrm{mph}$ or more over the posted speed limit & $2.93[2.62-3.24]$ \\
Making an illegal turn & $3.25[2.96-3.54]$ \\
Failing to move over for a bicyclist, pedestrian, or emergency vehicle & $3.48[3.16-3.80]$ \\
Failing to stop at a crosswalk for one or more pedestrians & $3.90[3.62-4.18]$ \\
Failing to take appropriate action to avoid a hazard & $3.99[3.76-4.23]$ \\
Swerving off the road (at least two wheels off the road) & $4.13[3.88-4.38]$ \\
Swerving into the wrong lane (at least two wheels in opposite lane) & $4.35[4.15-4.56]$ \\
Failing to stop at an intersection for a red light or stop sign & $4.42[4.21-4.64]$ \\
Traveling in the wrong direction on a one-way road & $4.66[4.51-4.81]$ \\
Moving into a lane (traveling in the same direction) occupied by another vehicle (leading to a near collision or collision) & $4.71[4.58-4.84]$ \\
Hitting a vehicle, pedestrian, bicyclist, or object & $4.96[4.90-5.02]$ \\
\hline
\end{tabular}

Note Numbers in brackets are $95 \%$ confidence intervals of the means

errors such as failing to yield to a vehicle with the right of way. Distinguishing the minor errors from the serious errors was important, because it was anticipated that the serious errors would be the primary basis for participants' assessments of the safeness of their driving and their confidence in their ability to drive safely while distracted.

Table 2 presents the serious and minor driving errors made in the cell-phone and control groups. A comparison of the errors in the two groups revealed that cell-phone participants made significantly more serious errors $t(98)=2.72, p=.008, d$ $=.55$, but not more minor errors, $t(98)=.68, p>.250, d=.14$, than control participants.

Participants' assessments of the safeness of their driving and their estimations of the number of driving errors they made in the cell-phone and control groups are also presented in Table 2. Cell-phone participants were expected to perceive their driving to be less safe and to report they made more errors because of the widespread expectation that cell phones impair driving performance. Somewhat surprisingly, cellphone participants did not remember making more serious errors, $t(98)=.67, p>.250, d=.13$, or more minor errors than control participants, $t(98)=1.33, p=.185, d=.27$. However, as anticipated, cell-phone participants perceived that they drove less safely than control participants, $t(98)=$ $4.97, p=.001, d=.99$. The groups also did not differ in terms of their perceptions of their general ability to dive safely while distracted following the simulator session, $t(98)=1.67, p=$ $.097, d=.34$.

\section{Relation between perceived and actual driving safeness}

Analyses were performed to examine whether participants' assessments of the safeness of their driving were based on awareness of the actual safeness of their driving (see Table 3). Control participants' assessments of their driving safety were negatively correlated with their serious driving errors, $r(49)=-.37$, and uncorrelated with their minor driving errors. Thus, control participants' assessments of the safeness of their driving decreased as the number of serious driving errors they made increased. By contrast, cell-phone participants' assessments of their driving safety tended to be positively correlated with their serious driving errors, $r(49)=.25$,

Table 2 Perceived and actual errors, self-assessments of driving, and perceived ability to drive safely while distracted as a function of talking versus not talking on a cell phone while driving $(\mathrm{N}=100)$ Note Numbers in brackets are $95 \%$ confidence intervals of the means

\begin{tabular}{llr}
\hline & Control & Cell-phone \\
\hline Serious driving errors & $4.38[3.80-4.95]$ & $5.86[4.96-6.76]$ \\
Minor driving errors & $1.66[1.17-2.15]$ & $1.92[1.36-2.48]$ \\
Perceived serious driving errors & $5.20[3.97-6.43]$ & $5.80[4.56-7.04]$ \\
Perceived minor driving errors & $2.06[1.48-2.64]$ & $2.63[2.03-3.23]$ \\
Perceived safeness of driving* & $1.10[.74-1.45]$ & $-0.27[-.67-0.14]$ \\
Perceived ability to drive safely while using a cell phone** & $3.41[3.01-3.79]$ & $2.93[2.50-3.30]$ \\
\hline
\end{tabular}

*7-point scale anchored by " $-3 "=$ not at all safe and " $+3 "=$ highly safe

**7-point scale anchored by "1" = not at all capable and "7" = highly capable 
Table 3 Correlations between actual and perceived errors, and memory for errors, self-assessments of driving, and perceived ability to drive safely while distracted as a function of talking versus not talking on a cell phone while driving ( $N=50$ in each group)

\begin{tabular}{|c|c|c|c|c|}
\hline & Actual serious errors & Actual minor errors & Perceived serious errors & Perceived minor errors \\
\hline \multicolumn{5}{|c|}{ Perceived safeness of driving } \\
\hline Cell phone & $.25^{*}$ & $-.32 * *$ & $-.39 * * *$ & $-.24 *$ \\
\hline \multirow[t]{2}{*}{ Control } & $-.37 * * *$ & -.07 & $-.70 * * *$ & -.17 \\
\hline & $\Delta * * *$ & & $\Delta^{*}$ & \\
\hline \multicolumn{5}{|c|}{ Perceived ability to drive safely while distracted } \\
\hline Cell phone & .13 & $-.24 *$ & $-.44 * * *$ & $-.34 * *$ \\
\hline \multirow[t]{2}{*}{ Control } & $-.32 * *$ & -.02 & $-.38 * * *$ & .05 \\
\hline & $\Delta^{* *}$ & & & $\Delta^{*}$ \\
\hline \multicolumn{5}{|c|}{ Memory for errors } \\
\hline Cell phone & .05 & $.29 * *$ & & \\
\hline \multirow[t]{2}{*}{ Control } & $.40 * * *$ & .13 & & \\
\hline & $\Delta^{*}$ & & & \\
\hline
\end{tabular}

* Significant at $p<.10$ level

$* *$ Significant at $p<.05$ level

$* * *$ Significant at $p<.01$ level

and uncorrelated with their minor driving errors. A comparison of these correlations revealed that self-assessments of driving safety were more accurate and better correlated with the actual driving errors in the control group than the cellphone group, $\mathrm{F}(1,96)=10.25, \mathrm{p}=.002, \eta^{2}=.10$. That is, cell-phone participants were less aware of the actual safeness of their driving than control participants.

Further evidence of cell-phone participants' lack of driving self-awareness comes from an analysis of their memory of their driving errors. Control participants' memory of their serious driving errors was positively correlated with their actual serious driving errors, $r(49)=.40$, but their memory of their minor driving errors was not correlated with their actual minor driving errors. The pattern suggests that control participants were cognizant of the serious errors they were making. In contrast, cell-phone participants' memory of their serious driving errors was not correlated with the actual number of serious errors they made, $r(49)=.05$. However, a comparison of the correlations found that the memory for serious driving errors did not significantly differ between cell-phone and control groups, $\mathrm{F}(1,96)=3.20, \mathrm{p}=.077, \eta^{2}=.03$.

\section{Predictors of confidence in driving ability}

The final set of analyses focused on the predictors of participants' general confidence in their ability to drive safely while distracted. From Table 3 it is apparent that both control and cell-phone participants' confidence in their ability to drive safety while distracted was negatively correlated with their perceived serious driving errors. However, only control participants' confidence in their ability to drive safely while distracted was grounded in the actual safeness of their driving.
The confidence of control participants was negatively correlated with their serious driving errors, $r(49)=-.32$, whereas the confidence of cell-phone participants was uncorrelated with their serious driving errors, $r(49)=.13$. The control and cell-phone groups differed in the relationship between the assessments of their ability to drive safely while distracted and their serious driving errors, $F(1,96)=5.04, p=.027, \eta^{2}=.05$.

\section{Discussion}

Cell-phone use not only impaired the safeness of participants' driving in our study, it impaired their awareness of the safeness of their driving. The driving safety assessments and memory of driving errors of cell-phone participants were uncorrelated with the actual errors they made. When drivers are talking on a cell phone, they are often unaware of the inconsistencies in their speed, their weaving across lanes, and their near misses with other vehicles. As a consequence, they may persist in believing that they can safely talk or text on a cell phone behind the wheel.

The study furthers our understanding of the impact of secondary task engagement on the self-regulation of performance. Taking on multiple tasks simultaneously can be physically taxing and stressful, and contribute to poorer primarytask performance. Our findings indicate that an additional cost of multitasking is a decrease in the performance monitoring that is essential to self-regulation and self-knowledge (e.g., Baumeister, Heatherton, \& Tice, 1994). Because multitaskers are less self-aware, they may be less apt to make the adjustments necessary to bring their performance in line with their goals (e.g., Carver \& Scheier, 1998) and less likely to acquire 
the self-knowledge of their shortcomings and abilities that is essential to decision making and improvement.

Importantly, these findings cast doubt on speculation that drivers routinely self-regulate their multitasking activities, such as talking on a cell phone, based upon their real-time driving performance (Young \& Regan, 2007). Because cellphone drivers are less aware of their errors, they are less apt to self-regulate their cell-phone usage based on the real-time demands of driving. Alarmingly, the cell-phone drivers who made the most errors exhibited a pattern similar to the fictional cartoon character Mr. Magoo, who was blithely unaware of his driving impairments.

The research also increases our understanding of the motivations underlying multitasking and the usage of cell phones while driving. As we discussed previously, people tend to downplay the risks of multitasking, in part, because of inflated views of their capacities (Sanbonmatsu et al., 2015). The present findings suggest that one of the contributors to overconfidence in the ability to multitask is diminished self-awareness. People are often unaware of the detrimental effects of doing many things at once on their performance. Consequently, they may persist in believing that they are capable of performing multiple tasks simultaneously with minimal cost. Although cell-phone participants in our study made more errors than controls, they were not aware of them. As a result, their assessments of their general driving ability were unrelated to their unsafe driving. In contrast, control participants' estimations of their ability to drive safely while distracted were significantly associated with the actual serious errors they made on the simulator.

An examination of the group means reveals that the mean perceived serious errors of cell-phone participants $(M=5.86)$ was very close to their actual mean serious errors $(M=5.80)$. This might suggest that cell-phone participants' recollections of their driving errors were relatively accurate. However, as we reported above, individual cell-phone participants' estimations of their serious errors were uncorrelated $(r=.05)$ with their actual serious errors. In contrast, the perceived and actual serious errors of control participants were highly positively correlated $(r=.40)$. Thus, it is clear from the correlational analyses that cell-phone participants were largely guessing about the driving errors they made and that the similarity of the group means appears to be happenstance.

People generally acknowledge that cell phone use impairs driving performance (Sanbonmatsu et al., 2015). This expectation is likely to have led cell-phone participants to assess their driving safety less favorably than controls and to estimate a greater number of driving errors. However, participants who talked on a cell phone really had little idea of how well or poorly they were driving. Multitasking is often attention demanding to the point that people are unable to monitor themselves and the circumstances affecting their behavior. As a consequence, their assessments and accounts of their performance may often be specious.
Acknowledgments This work was supported by a grant from the Mountain Plains Consortium, a regional University Transportation Center (UTC) sponsored by the United States Department of Transportation, Research and Innovative Technology Administration. The authors thank Shazia Ahmad, Erica Armstrong, Jennifer Buttrick, Daniel Cosentino, Laura Creamer, Ciera Figge, Brooke Rowley, Tarra Sliwa, and Jennifer Webb for their assistance in conducting the study.

Author note D.M. Sanbonmatsu and D.L. Strayer developed the study concept. All authors contributed to the experimental design. Simulator programming, testing, and data collection were performed by F. Biondi, A.A. Behrends, and S.M. Moore. All authors contributed to the data analysis and interpretation. D.M Sanbonmatsu and D.L. Strayer contributed equally to the writing of the manuscript and the order of authorship was determined alphabetically. All authors approved the final version of the manuscript for submission.

\section{References}

Baumeister, R. F., Heatherton, T. F., \& Tice, D. M. (1994). How and why people fail at self-regulation. San Diego: Academic Press References.

Carver, C. S., \& Scheier, M. F. (1998). On the self-regulation of behavior. New York: Cambridge University Press.

Crenshaw, D. (2008). The Myth of multitasking: How "Doing It ALL" Gets Nothing Done. San Francisco: Jossey-Bass.

Federal Highway Administration (2012). Manual on Uniform Traffic Control Devices - 2009 Edition with Revision Numbers 1 and 2. http://mutcd.fhwa.dot.gov/kno_2009r1r2.htm

Glassbrenner, D. (2005). Traffic safety facts research note: Driver cell phone use in 2005 - Overall results. DOT HS 809 967. Washington, DC: National Center for Statistics and Analysis, National Highway Traffic Safety Administration.

McEvoy, S. P., Stevenson, M. R., McCartt, A. T., Woodward, M., Haworth, C., Palamara, P., \& Cercarelli, R. (2005). Role of mobile phones in motor vehicle crashes resulting in hospital attendance: A case-crossover study. British Medical Journal, 331, 428-433.

Moore, S. M., Behrends, A. A., Mazur, D., and Sanbonmatsu, D. M. (2014). When do people bet on their selves? The role of global vs. specific self-concepts in decision-making. Manuscript submitted for publication.

Redelmeier, D. A., \& Tibshirani, R. J. (1997). Association between cellular-telephone calls and motor vehicle collisions. The New England Journal of Medicine, 336, 453-458.

Sanbonmatsu, D. M., Strayer, D. L., Medeiros-Ward, N., Behrends, A. A., and Watson, J. M. (2015). Why drivers use cell phones and why they support legislation to restrict this practice. Manuscript submitted for publication.

Sanbonmatsu, D. M., Strayer, D. L., Medeiros-Ward, N., \& Watson, J. M. (2013). Who multi-tasks and why? Multi-tasking ability, perceived multi-tasking ability, impulsivity, and sensation seeking. PLOS ONE, 8(1), e54402. doi:10.1371/journal.pone.0054402

Sayette, M. A., \& Griffin, K. M. (2011). Self-regulatory failure and addiction. In K. D. Vohs \& R. F. Baumeister (Eds.), Handbook of SelfRegulation: Research, Theory, and Applications (2nd ed., pp. 505521). New York: Guilford Press.

Sayette, M. A., Schooler, J. W., \& Reichle, E. D. (2010). Out for a smoke: The impact of cigarette craving on zoning-out during reading. Psychological Science, 20, 747-752.

Strayer, D. L., \& Drews, F. A. (2007). Cell-phone induced inattention blindness. Current Directions in Psychological Science, 16, 128-131.

Strayer, D. L., Drews, F. A., \& Johnston, W. A. (2003). Cell phone induced failures of visual attention during simulated 
driving. Journal of Experimental Psychology: Applied, 9, 2352.

Strayer, D. L., Watson, J. M., \& Drews, F. A. (2011). Cognitive distraction while multitasking in the automobile. In B. Ross (Ed.), The Psychology of Learning and Motivation (Vol. 54, pp. 29-58).
Young, K., \& Regan, M. (2007). Driver distraction: A review of the literature. In I. J. Faulks, M. Regan, M. Stevenson, J. Brown, A. Porter, \& J. D. Irwin (Eds.), Distracted Driving (pp. 379-405). Sydney: Australasian College of Road Safety. 